\title{
Streptococcus pyogenes Meningitis in Children: Report of Two Cases and Literature Review
}

\author{
Mariana V. Arnoni, Eitan N. Berezin, Marco A.P. Sáfadi, Flávia J. Almeida and Cláudia R.C. Lopes \\ Pediatric Infectious Diseases Service, Santa Casa de São Paulo Hospital; São Paulo, SP, Brazil
}

\begin{abstract}
Streptococcus pyogenes meningitis (SPM) occurs sporadically, even with the increase of invasive streptococcal disease observed in the past years. We reported two cases of SPM in infants to alert pediatricians for the possibility of this agent as a cause of meningitis in previously healthy children.

Key-Words: Streptococcus pyogenes, meningitis, children.
\end{abstract}

Streptococcus pyogenes is an agent rarely associated to meningitis and corresponds to $0.2-1 \%$ of the cases [1,2], usually as a contiguous infection site, such as otitis, sinusitis and mastoiditis [1].

Since 1980s, there was a great increase in the incidence of invasive streptococcal disease [3], which many times is fulminating, with mortality higher than $30 \%$ and also affecting healthy individuals [4]. Despite of this, the rates of SPM remain low in adult and pediatric population [5]. Through a review of the literature within the last 10 years, we found 13 cases descriptions of SPM in pediatric patients which are described in Table 1.

We reported 2 cases of previously healthy children that developed SPM. One of them presented a clinically favorable evolution and the other died. The purpose of this study is to emphasize that this agent is also a cause of meningitis in healthy children.

\section{Case Reports}

Case 1

A previously healthy 5 -year-old child that presented to our service with fever, vomiting, ear ache and headache started one day before. During initial examination, the patient appeared to be in a toxic condition, with hyperemia of the right tympanic membrane, and her neck was stiff. The patient was submitted to a lumbar puncture and the cerebrospinal fluid (CSF) was indicative of bacterial meningitis and therapy with ceftriaxone and dexamethasone was immediately instituted.

Laboratory investigation revealed a white blood cell count of 30,200 leukocytes $/ \mu \mathrm{L}$ with $96 \%$ neutrophils, ESR $90 \mathrm{mM}$, CSF with 1,050 cells $/ \mathrm{mm}^{3}$ ( $46 \%$ neutrophils), protein of 150 $\mathrm{mg} / \mathrm{dL}$, glucose of $22 \mathrm{mg} / \mathrm{dL}$, negative Gram stain and negative latex test. Blood culture was negative and CSF culture identified SP and the strain was sensitive to penicillin. Computed tomography demonstrated signs of right mastoiditis.

Received on 3 January 2007; revised 20 May 2007.

Address for correspondence: Dr. Mariana Volpe Arnoni. Rua Jesuíno Arruda 318 apto.121 - Itaim Bibi - CEP 04532-080. São Paulo SP - Brasil. Phone/Fax: (55+11) 31684517. E-mail: volpearnoni@uol.com.br.

The Brazilian Journal of Infectious Diseases 2007;11(3):375-377. (C) 2007 by The Brazilian Journal of Infectious Diseases and Contexto Publishing. All rights reserved.
The antibiotic regimen was changed to penicillin. After two days, the patient developed right peripheral facial palsy, but showed improvement of general condition. A meringotomy was performed on the right with placement of a Sheppard's ventilation tube.

The patient was kept on antimicrobial therapy for 10 days and showed a very favorable clinical evolution, despite maintaining peripheral facial palsy on hospital discharge. During ambulatory follow-up, CT and audiometry were normal and there was total regression of the facial palsy.

\section{Case 2}

A previously healthy 3-month-old child that arrived at our service with a one day history of fever and irritability. On initial examination the patient was in a poor condition, with grey skin, poor muscle tone and bulging of the fontanelle. The patient was diagnosed with varicella approximately 20 days earlier and had favorable clinical evolution.

The patient was submitted to a lumbar puncture and the CSF indicated signs of bacterial meningitis with 1,369 cells/ $\mathrm{mm}^{3}$ (90\% neutrophils), protein of $150 \mathrm{mg} / \mathrm{dL}$, glucose of 10 $\mathrm{mg} / \mathrm{dL}$ and negative Gram stain. Treatment was initiated with ceftriaxone and dexamethasone, in addition to clinical support measures. Approximately one hour after admission, the patient evolved with septic shock and cardiorespiratory arrest and did not respond to reanimation procedures. SP sensitive to penicillin was isolated from CSF.

\section{Discussion}

The occurrence of sporadic cases of SPM has been documented in the literature and has not suffered increase in its incidence in the last decades, differently from what has been described about invasive streptococcal disease [5].

The pathogenesis of SPM has not been entirely explained. Generally, it is not present as a complication of the invasive disease and there is frequently an association with contiguous infectious foci of the upper respiratory tract. Furthermore, some risk factors have been described: neurosurgery, skull fractures, CSF leaks, infection or colonization of the upper respiratory tract and skin lesions (impetigo, varicella and erysipelas) [1,5]. However, SPM also occurs in healthy individuals that do not have predisposing conditions and do not present clear evidences of a contiguous focus [3]. In table 1 , we observed that $66 \%$ of the reported cases presented a predisposing risk factor. 
Table 1. Cases of SPM from 1996 to 2006

\begin{tabular}{|c|c|c|c|c|c|c|c|c|}
\hline $\begin{array}{c}\text { Case } \\
\text { (n) }\end{array}$ & Age & Risk factor & Treatment & Evolution & Culture & Year & Country & Ref \\
\hline 1 & $3 \mathrm{~m}$ & Infected BCG scar & CTX & Good & $\begin{array}{l}\text { Blood/CSF/ } \\
\text { BCG scar }\end{array}$ & 2000 & Engl & 6 \\
\hline 2 & $2 \mathrm{~m}$ & Infected haemangioma & CTX/Vanco/Aci & Encephalomalacia & $\mathrm{CSF}$ & 2003 & Can & 7 \\
\hline 3 & $2 \mathrm{a}$ & No & Amp/CRO/Clo & Good & CSF & 2004 & Ind & 8 \\
\hline 4 & $10 \mathrm{a}$ & Submandibular Abscess & Peni/CRO & Good & $\mathrm{CSF}$ & 2004 & Ind & 8 \\
\hline 5 & $2.5 \mathrm{a}$ & No & CRO/Vanco & Death & CSF/Blood & 1998 & USA & 4 \\
\hline 6 & $4 \mathrm{a}$ & Cochlear implantation & $\mathrm{CRO}$ & Neurological delay & CSF & 2002 & Can & 9 \\
\hline 7 & $11 \mathrm{a}$ & Otitis & CTX & Paralysis of 3rd cranial pair & Blood & 2001 & Ger & 11 \\
\hline 8 & $18 \mathrm{~d}$ & No & Peni/CRO & Neurological delay thrombosis & $\mathrm{CSF}$ & 1998 & $\mathrm{Br}$ & 10 \\
\hline 9 & $6 \mathrm{a}$ & Otitis & Peni & Good & $\mathrm{CSF}$ & 2000 & Ger & 3 \\
\hline 10 & $15 \mathrm{a}$ & Occipital skull & $\mathrm{CRO}$ & Good & CSF/Blood & 1999 & Swits & 5 \\
\hline 11 & $1 \mathrm{~m}$ & No & Broad ATB & Death & CSF/Blood & 1998 & USA & 11 \\
\hline 12 & $2 \mathrm{~m}$ & No & CTX/Amp & Good & $\mathrm{CSF}$ & 1998 & Isr & 12 \\
\hline 13 & $3 \mathrm{~m}$ & Varicella & CTX/Peni & Good & $\mathrm{CSF}$ & 2003 & Ger & 13 \\
\hline 14 & $5 \mathrm{a}$ & Otitis & Peni/CRO & Good & $\mathrm{CSF}$ & 2002 & $\mathrm{Br}$ & \\
\hline 15 & $3 \mathrm{~m}$ & Varicella & $\mathrm{CRO}$ & Death & CSF & 2002 & $\mathrm{Br}$ & \\
\hline
\end{tabular}

$\mathrm{N}=$ number. $\mathrm{CTX}=$ cefotaxime; Vanco=vancomycin; Aci=acyclovir, Amp=ampicillin; CRO=ceftriaxone, Clo=cloramphenicol, Peni=penicillin; Broad $\mathrm{ATB}=$ broad spectrum antibiotic. Engl=England; Can=Canada; Ind=India; Ger=Germany; Swits=Switzerland; Br=Brazil; Isr=Israel.

Despite the fact that upper respiratory tract colonization or infection by SP is very common during infancy, the rates of meningitis correspond to less than $1 \%$ of the bacterial meningitis. This suggests that direct meningeal invasion is not the likely cause of the infection and that there may be involvement of a genetic predisposition. SP also seems to have little affinity for the central nervous system $[2,4]$.

SPM may affect any age group. In our research, we observed cases from neonates to 15 -year-old adolescents. The occurrence of outbreaks has been described in neonatal units and onphalitis is the most common primary infection site [10].

In older children and adults, contiguous infection foci are most frequently associated with the disease, especially acute otitis media, although in many cases a primary contiguous focus is not identified [3]. In the first case that we presented, the patient had acute otitis media as a risk factor. This data is in agreement with other literature cases, showing upper respiratory tract infections (otitis, pharyngitis, sinusitis, rhinitis and tonsillitis) as important risk factors.

In the second case, the patient had a recent history of varicella, which was also described as a risk factor.

Infectious complications of varicella are described in $39 \%$ of the cases and SP is the main agent involved. However, post-varicella SPM has rarely been reported and it seems to be related to skin lesions [13].

The clinical presentation of SPM is not different from the other bacterial meningitis and neither are the findings from the CSF analysis.

The antibiotic of choice for the treatment of SPM is penicillin and there have been no reports of resistance of this agent to this drug. Ceftriaxone corresponds to the alternative for cases in which the patient is allergic to penicillin. The association of penicillin with clindamycin is the recommended treatment for Streptococcal Toxic-Shock Syndrome and necrotizing soft tissue infections because clindamycin does not suffer the effects of the great inoculums and has better action upon the streptococcic exotoxins [4].

Our cases were initially treated with ceftriaxone, which constitutes the recommended empirical treatment of meningitis cases in our environment. After the etiologic diagnosis was made, in the first case, the treatment was changed to penicillin and the total treatment duration was 10 days. In reviewing the literature, we also observed that the initial empirical treatment constitutes the use of $3^{\text {rd }}$ generation cephalosporins. In some cases, even after the etiological diagnosis, the antibiotic treatment was not changed to penicillin, despite SP having a preserved sensitivity to penicillin.

In SPM during infancy, the evolution to neurological complications and sequelae are described in $60 \%$ of the cases, with a mortality rate of up to $12 \%[1,3]$. Chow \& Muder reported a global lethality of 5\% and sequelae in $46 \%$ of the cases in a review conducted between 1981 and 1991 [14].

The second case we presented had a fulminating evolution, with death occurring within the first hours after hospital admittance, even with symptom onset occurring only one day before hospital care. This demonstrates how severe this infection can be. In the literature, 3 cases of death were found, lethality was $20 \%$ and evolution to sequelae in $26.7 \%$ of the cases.

\section{Conclusion}

We conclude that, despite its sporadic occurrence, SP should be considered as a cause of meningitis in previously healthy infants, because of its frequent association with complications, sequelae and death. 


\section{Acknowledgments}

We are grateful to the Support Center for Scientific Publications of Santa Casa de São Paulo - Faculty of Medical Sciences for the editorial assistance.

\section{References}

1. Steppberger K., Adams I., Deutscher J., et al. Meningitis in a girl with recurrent otitis media caused by Streptococcus pyogenes-otitis media has to be treated appropriately. Infection 2001;29:286-8.

2. Van de Beek D., de Gans J., Spanjaard L., et al. Group a Streptococcal meningitis in adults: report of 41 cases and a review of the literature. Clin Infect Dis 2002;34:e32-e6.

3. Berner R., Silke H., Gordjani N., Brandis M. Streptococcus pyogenes meningitis: report of a case and review of the literature. Eur J Pediatr 2000; $159: 527-9$

4. Shetty A.K., Frankel L.R., Maldonado Y., et al. Group A streptococcal meningitis: report of a case and review of literature since 1976. Pediatr Emerg Care 2001;17:430-4.

5. Sommer R., Rohner P., Garbino J., et al. Group A beta-hemolytic streptococcus meningitis: clinical and microbiological features of nine cases. Clin Infect Dis 1999;29:929-31.

6. Melzer M., Joashi U., Murdoch I., Gransden W. Group A streptococcal meningitis with an infected BCG scar. J R Soc Med 2000;93:641.
7. Rezvani M., Yager J.Y., Hartfield D.S. Group A streptococcal meningitis as a complication of an infected capillary haemangioma. Eur J Pediatr 2004; $163: 19-21$

8. Mathur P., Arora N.K., Kapil A., Das B.K. Streptococcus pyogenes meningitis. Indian J Pediatr 2004;71:423-6.

9. Pettersen G., Ovetchkine P., Tapiero B. Group A streptococcal meningitis in a pediatric patient following cochlear implantation: report of the first case and review of the literature. J Clin Microbiol 2005; 43:5816-5818

10. Krebs V.L., Chieffi L.N., Jurfest M.E., et al. Meningite Neonatal por Streptococcus pyogenes e Trombose do Seio Sagital. Arq Neuropsiquiatr 1998;56:829-832.

11. Givner L.B. Invasive disease due to group A beta-hemolytic streptococci: continued occurrence in children in North Carolina. South Med J 1998;91:333-337.

12. Moses A.E., Beeri M., Engelhard D. Group A streptococcal meningitis: report of two cases. J Infect 1998;36:116118 .

13. Brandt C.M., Kitz R., Lutticken R., Brade V. Streptococcus pyogenes meningitis complicating varicella in a 3-month-old child. Scand J Infect Dis 2003;35:876-878.

14. Chow J.W., Muder R.R. Group A streptococcal meningitis. Clin Infect Dis 1992;14:418-421. 